EESTI NSV TEADUSTE AKADEEMIA TOIMETISED.

FOOSIKA * MATEMAATIKA

ИЗВЕСТИЯ АКАДЕМИИ НАУК ЭСТОНСКОП ССР. ФИЗИКА * МАТЕМАТИКА

PROCEEDINGS OF THE ACADEMY OF SCIENCES OF THE ESTONIAN SSR.

PHYSICS * MATHEMATICS

$1985,34,2$

681.511 .22

Ю. НУРГЕС

\title{
ЛАГЕРРОВА МОДЕЛЬ «ВХОД-ВЫХОД»
}

D. NURGES. LAGUERRE'I SISEND-VALJUNDMUDEL

O. NURGES. INPUT-OUTPUT LAGUERRE MODEL

\section{(Представил Н. Алумяэ)}

Введение. Модели типа «вход-выход» наиболее подходящие для решения задач идентификации. Для сглаживания и сжатия входных и выходных сигналов динамической системы используется успешно их разложение по ортогональным многочленам Лагерра $\left[{ }^{1-3}\right]$. В $\left.{ }^{1}\right]$ определены соотношения между коэффициентами разложения входных и выходных сигналов скалярной линейной системы через ее разностное уравнение $n$-го порядка. В $\left[{ }^{3}\right]$ предложены лагерровы уравнения состояния, связывающие коэффициенты разложения входных воздействий и выходных переменных через абстрактный вектор состояния лагерровой модели.

В данной статье выводится лагеррова модель типа «вход-выход» многомерной дискретной системы, связывающая непосредственно коэффициенты разложения входных и выходных сигналов системы. Найдены формулы перехода от разностного уравнения системы к лагерровой модели «вход-выход» и обратно.

Подстановка задачи. Пусть многомерная дискретная система представлена разностным уравнением $n$-го порядка, т. е. моделью типа «вход-выход»

$$
\begin{gathered}
A_{n} y(t+n)+A_{n-1} y(t+n-1)+\ldots+A_{0} y(t)+ \\
+B_{n-1} u(t+n-1)+\ldots+B_{0} u(t)=0, \\
y(\tau)=u(\tau)=0, \quad \tau \leqslant n-1,
\end{gathered}
$$

где $u(t) \in R^{m}, y(t) \in R^{p}$ - векторы входа и выхода соответственно, матрицы $A_{0}, \ldots, A_{n}$ размерности $p \times p$ и $B_{0}, \ldots, B_{n-1}$ размерности $p \times m$, $n$ - порядок системы.

Разлагая дискретные последовательности входных воздействий $\{u(t)\}$ и выходных переменных $\{y(t)\}, t=0,1,2, \ldots$ в ряд Фурье по разностным многочленам Лагерра

$$
\psi_{k}(t)=\sqrt{1-\xi^{2}} \sum_{j=0}^{k}(-1)^{k+j}\left(\begin{array}{l}
k \\
j
\end{array}\right)\left(\begin{array}{c}
t+k-j \\
k
\end{array}\right) \xi^{t+k-2 j},
$$

где $\xi-$ постоянная Лаггера, $\xi \in(-1,1),\left(\begin{array}{l}k \\ j\end{array}\right)$ - биномиальный коэффициент, получим дискретные последовательности лагерровых коэффициентов $\left\{u_{k}\right\}$ и $\left\{y_{k}\right\}, k=0,1,2, \ldots$.

Учитывая обстоятельство, что связь между коэффициентами разло- 
жения $u_{k}$ и $y_{k}$ представима линейной динамической системой порядка $n$ $\left[{ }^{3}\right]$, ищем лагеррову модель типа «вход-выход» в виде

$$
F_{n} y_{k+n}+\ldots+F_{0} y_{k}+G_{n} u_{k+n}+\ldots+G_{0} u_{k}=0,
$$

где $y_{k} \in R^{p}, u_{k} \in R^{m}, \quad$ матрицы $F_{0}, \ldots, F_{n}$ размерности $p \times p$ и $G_{0}, \ldots, G_{n}$ размерности $p \times m$.

Разностные многочлены Лагерра. Любая дискретная функция $f(t)$, $t=0,1,2, \ldots$ разложима в ряд Фурье по многочленам $\psi_{k}(t)$ в виде

$$
f(t)=\sum_{k=0}^{\infty} f_{k} \psi_{k}(t)
$$

где коэффициенты разложения $f_{k}$ определяются соотношением

$$
f_{k}=\sum_{t=0}^{\infty} f(t) \psi_{k}(t)
$$
$\left[{ }^{3}\right]$ :

Вычисление многочленов $\psi_{k}(t)$ облегчают рекуррентные формулы

$$
\begin{gathered}
\psi_{0}(0)=\sqrt{1-\xi^{2}} ; \\
\psi_{0}(t+1)=\xi \psi_{0}(t), \\
\psi_{k}(t+1)=\xi \psi_{k}(t)+\left(1-\xi^{2}\right) \sum_{i=0}^{k-1}(-\xi)^{k-i-1} \psi_{i}(t) .
\end{gathered}
$$

Учитывая свойство (5), получим следующее важное соотношение

$\xi \psi_{k}(t+j)+\psi_{k+1}(t+j)=\psi_{k}(t+j-1)+\xi \psi_{k+1}(t+j-1), j=1,2, \ldots$.

Многократное применение соотношения (6) дает общую формулу смещения во временной области

$$
\sum_{r=0}^{j}\left(\begin{array}{l}
j \\
r
\end{array}\right) \xi^{j-r} \psi_{k+r}(t+j)=\sum_{r=0}^{j}\left(\begin{array}{l}
j \\
r
\end{array}\right) \xi^{r} \psi_{k+r}(t), j=1,2, \ldots
$$

Вывод лагерровой «вход-выход» модели. Умножая уравнение (1) на $\psi_{k}(t)$ и суммируя по $t=0,1,2, \ldots$, получим соотношения между векторами коэффициентов разложения дискретных функций $u(t)$ и $y(t)$ по разностным многочленам Лагерра

$$
A_{n} y_{k}^{(n)}+\ldots+A_{1} y_{k}^{(1)}+A_{0} y_{k}+B_{n-1} u_{k}^{(n-1)}+\ldots+B_{1} u_{k}^{(1)}+B_{0} u_{k}=0,
$$

где

$$
\begin{aligned}
& y_{k}^{(j)}=\sum_{t=0}^{\infty} y(t+j) \psi_{k}(t), \quad j=1, \ldots, n, \\
& u_{k}^{(i)}=\sum_{t=0}^{\infty} u(t+i) \psi_{k}(t), \quad i=1, \ldots, n-1 .
\end{aligned}
$$

По формуле (3)

$$
y(t+j)=\sum_{l=0}^{\infty} y_{l} \psi_{l}(t+j)
$$

На основании формулы смещения (7) имеем 


$$
\begin{gathered}
\sum_{r=0}^{j}\left(\begin{array}{l}
j \\
r
\end{array}\right) \xi^{r} y_{k+r}^{(j)}=\sum_{t=0}^{\infty} y(t+j) \sum_{r=0}^{j}\left(\begin{array}{l}
j \\
r
\end{array}\right) \xi^{r} \psi_{k+r}(t)= \\
=\sum_{t=0}^{\infty} y(t+j) \sum_{r=0}^{j}\left(\begin{array}{l}
j \\
r
\end{array}\right) \xi^{j-r} \psi_{k+r}(t+j)= \\
=\sum_{r=0}^{j}\left(\begin{array}{l}
j \\
r
\end{array}\right) \xi^{j-r} y_{k+r}-\sum_{\tau=0}^{j-1} y(\tau) \sum_{r=0}^{j}\left(\begin{array}{l}
j \\
r
\end{array}\right) \xi^{j-r} \psi_{k+r}(\tau) .
\end{gathered}
$$

Рассматриваем сумму

$$
S=\sum_{j=0}^{n} A_{j} \sum_{r=0}^{n}\left(\begin{array}{l}
n \\
r
\end{array}\right) \xi^{r} y_{k+r}^{(j)}+\sum_{i=0}^{n-1} B_{i} \sum_{r=0}^{n}\left(\begin{array}{l}
n \\
r
\end{array}\right) \xi^{r} u_{k+r^{(i)}} .
$$

Учитывая свойство биномиальных коэффициентов

$$
\left(\begin{array}{l}
n \\
r
\end{array}\right)=\sum_{\alpha=0}^{\beta}\left(\begin{array}{l}
\beta \\
\alpha
\end{array}\right)\left(\begin{array}{c}
n-\beta \\
r-\alpha
\end{array}\right), \quad \beta=1, \ldots, n
$$

и соотношение (9), получим при нулевых начальных условиях $y(\tau)=0$, $\tau=0, \ldots, n-1$

$$
\sum_{r=0}^{n}\left(\begin{array}{c}
n \\
r
\end{array}\right) \xi^{r} y_{k+r}^{(j)}=\sum_{h=0}^{n-j} \sum_{r=0}^{j}\left(\begin{array}{c}
n-j \\
h
\end{array}\right)\left(\begin{array}{l}
j \\
r
\end{array}\right) \xi^{j+h-r} y_{k+h+r}
$$

Аналогичное соотношение получим при нулевых начальных условиях $u(\tau)=0, \tau=0, \ldots, n-1$ для коэффициентов разложения входных воздействий.

На основании (10) сумма $S$ равняется

$$
\begin{aligned}
& S=\sum_{j=0}^{n} \sum_{h=0}^{n-j} \sum_{r=0}^{j} A_{j}\left(\begin{array}{c}
n-j \\
h
\end{array}\right)\left(\begin{array}{l}
j \\
r
\end{array}\right) \xi^{j+h-r} y_{k+h+r}+ \\
& +\sum_{i=0}^{n-1} \sum_{h=0}^{n-i} \sum_{r=0}^{i} B_{i}\left(\begin{array}{c}
n-i \\
h
\end{array}\right)\left(\begin{array}{l}
i \\
r
\end{array}\right) \xi^{i+h-r} u_{h+h+r},
\end{aligned}
$$

а по (8) $S=0$.

Значит, соотношение (11) представляет собой лагеррово уравнение «вход-выход»в неявном виде. Переписав уравнение (11) в явном виде $(2)$, получим следующие выражения для матриц $F_{i}$ и $G_{i}, i=0, \ldots, n$

$$
\begin{aligned}
& F_{i}=\sum_{j=0}^{n} \sum_{r=0}^{j}\left(\begin{array}{c}
n-j \\
i-r
\end{array}\right)\left(\begin{array}{l}
j \\
r
\end{array}\right) \xi^{i+j-2 r} A_{j}, \\
& G_{i}=\sum_{j=0}^{n-1} \sum_{r=0}^{j}\left(\begin{array}{c}
n-j \\
i-r
\end{array}\right)\left(\begin{array}{l}
j \\
r
\end{array}\right) \xi^{i+j-2 r} B_{j} .
\end{aligned}
$$

Отметим, что по определению биномиальных көэффициентов выражения (12) и (13) включают только те составляющие, при которых $n-j \geqslant i-r$ и $j \geqslant r$.

Заключение. Определена лагеррова модель типа «вход-выход» многомерной дискретной системы, а также ее связь с разностным уравнением $n$-го порядка.

Лагеррова модель «вход-выход» позволяет решить задачу идентификации посредством дискретного разложения Лагерра по следующей схеме: во-первых, найдем векторы коэффициентов разложения $\left\{u_{k}\right\}$ и 
$\left\{y_{k}\right\}, k=0, \ldots, N ; N \geqslant 2 n$, во-вторых, обыкновенными методами идентификации определим матрицы $F_{i}$ и $G_{i}, i=1, \ldots, n$, в-третьих, по формулам $(12),(13)$ вычислим матрицы $A_{0}, \ldots, A_{n}$ и $B_{0}, \ldots, B_{n-1}$. Эта схема идентификации существенно отличается от предложенной в $\left[{ }^{1}\right]$ процедуры и дает более хорошие результаты.

\section{Л ИТЕРАТУРА}

1. Paraskevopoulos, P. N., King, R. E. In: Identification and System Parameter Estimation, IV IFAC Symposium. Moscow, Part 2, 536-543, 1976.

2. Hwang, R.-Y., Shih, Y.-P. Int. J. Control, 37, № 3, 615-622 (1983).

3. Нургес Ю., Яаксоо Ю. Автоматика и телемеханика, № 12, 28-30 (1981).

Ннститут кибернетики

Академии наук Эстонской ССР
Поступила в редакцию 27/III 1984 